\title{
2-TYPE SURFACES IN A HYPERSPHERE
}

\author{
Th. Hasanis and Th. Vlachos
}

\section{Introduction}

Submanifolds of finite type were introduced by B.Y. Chen in [4]. This class of submanifolds is extremely large, including minimal submanifolds of the Euclidean space and minimal submanifolds of hyperspheres. In the last decade many researchers dealed with the study of finite type submanifolds.

In the present paper we study finite type surfaces in a hypersphere. We will review the definition of finite type submanifolds. Let $M^{n}$ be an $n$-dimensional submanifold of the unit hypersphere $S^{m}$ of the $(m+1)$-dimensional Euclidean space $E^{m+1}$ equipped with the induced metric. Denote by $\Delta$ the Laplacian operator of $M^{n}$ with sign convention such that $\Delta=-d^{2} / d t^{2}$ on the real line $E$. This operator can be extended in a natural way to $E^{m+1}$-valued maps on $M^{n}$. The submanifold $M^{n}$ is said to be of finite $k$-type if the position vector $x$ admits the spectral decomposition

$$
x=x_{0}+x_{\imath_{1}}+\cdots+x_{\imath_{k}},
$$

where $x_{0}$ is a constant vector and $x_{\imath}(\jmath=1, \cdots, k)$ are non-constant $E^{m+1}$-valued maps on $M^{n}$ such that

$$
\Delta x_{i_{j}}=\lambda_{i_{j}} x_{i_{j}}, \quad \lambda_{\imath_{j}} \in R, \quad \lambda_{i_{1}}<\cdots<\lambda_{i_{k}} .
$$

If the constant vector $x_{0}$ is the center of the hypersphere $S^{m}$ then $M^{n}$ is said to be mass-symmetric in $S^{m}$.

In terms of finite type terminology the well known Takahashi's Theorem [14] asserts that the submanifold $M^{n}$ of $S^{m}$ is of 1-type if and only if it is minimal in $S^{m}$ or in a hypersphere of $S^{m}$. Moreover minimal submanifolds of $S^{m}$ are mass-symmetric in $S^{m}$. From this point of view mass-symmetric 2-type submanifolds in $S^{m}$ are the simplest submanifolds next to minimal submanifolds in $S^{m}$.

In [8] it was proved that a surface in $S^{3}$ is of 2-type if and only if it is a portion of a Riemannian product of two circles of different radii. Moreover in [9] it was proved that a hypersurface of $S^{m}$ is of 2-type if and only if the scalar curvature and the mean curvature are constants.

Received June 14, 1994. 
The following interesting problem has been posed in [5] by B.Y. Chen: Do there exist mass-symmetric 2-type surfaces which lie fully in an evendimensional hypersphere?

In [1], M. Barros and B. Y. Chen proved that a compact stationary masssymmetric 2-type surface in $S^{m}$ is flat and lies fully in $S^{5}$ or in $S^{7}$. Furthermore they proved that there exist no mass-symmetric 2-type surfaces which lie fully in $S^{4}$. After that O. J. Garay [7] showed that a mass-symmetric 2-type Chen surface in $S^{m}$, that is a surface with zero allied mean curvature vector ([3], p. 203), is flat and lies fully in $S^{3}$ or in $S^{5}$ or in $S^{7}$ unless it is pseudoumbilical.

In [12] Y. Miyata based on a powerfull result of R. Bryant [2] treated mass-symmetric 2-type surfaces $M^{2}(c)$ in $S^{m}$ with constant Gauss curvature $c$ and proved, among others, that: Let $M^{2}(c)$ be a mass-symmetric 2-type surface in $S^{m}$ with $0<\lambda_{1}<\lambda_{2}$ then, (i) there exists no such surface with $c<0$ and (ii) if $c \geqq 0$ and $M^{2}(c)$ lies fully in $S^{m}$ then $m$ is odd. Finally M. Kotani [11] showed that if $M$ is a compact mass-symmetric 2-type surface of genus zero which lies fully in $S^{m}$ then $m$ is odd.

In this paper we will discuss this problem. The paper is organized as follows. In the first paragraph we prove some basic lemmas that will be needed in the proofs of the main results. In the second paragraph we give some local results. Specifically we prove that: If $M$ is a mass-symmetric 2-type surface which lies fully in $S^{m}$ and $\operatorname{dim} N_{1} \leqq 2$, where $N_{1}$ stands for the first normal space of $M$ in $S^{m}$, then $m$ is odd unless $M$ is pseudoumbilical. In the third paragraph we prove global results for mass-symmetric 2-type surfaces in $S^{m}$. In particular we prove that: If $M$ is a complete mass-symmetric 2-type surface in $S^{m}$ with non-negative Gaussian curvature then $M$ is flat and $m$ is odd unless $M$ is pseudoumbilical. Finally in the last paragraph we prove that there exist no mass-symmetric 2-type surfaces which lie fully in $S^{6}$.

\section{Basic lemmas}

Let $M$ be a surface of the unit hypersphere $S^{m}$ of $E^{m+1}$ centered at the origin $O$. Denote by $H, A, D$ the mean curvature vector, the Weingarten map and the normal connection of $M$ in $S^{m}$ respectively. Moreover $\bar{H}, \bar{A}$ and $\bar{D}$ denote the corresponding quantities for $M$ in $E^{m+1}$. We choose a local orthonormal frame field $\left\{e_{1}, e_{2}, \cdots, e_{m+1}\right\}$ on $M$ such that $e_{1}, e_{2}$ are tangent to $M$, $e_{3}=x$ and $H=|H| e_{4}$, where $x$ is the position vector field with respect to the center $O$. Denote by $\omega_{1}, \omega_{2}$ the dual frame of $e_{1}, e_{2}$. The connection form $\omega_{A B}, A, B=1,2, \cdots, m+1$ is given by $\omega_{A B}(X)=\left\langle\bar{\nabla}_{X} e_{A}, e_{B}\right\rangle$, where $\bar{\nabla}$ stands for the usual Riemannian connection of $E^{m+1}$. By Cartan's Lemma we have

$$
\omega_{i \alpha}=\sum_{j=1}^{2} h_{i j}^{\alpha} \omega_{j}, \quad \alpha \geqq 3, \imath=1,2,
$$

where $h_{\imath \jmath}^{\alpha}$ are the coefficients of the second fundamental form. 
The surface $M$ is said to be pseudoumbilical at the point $p \in M$ if the Weingarten map $A_{4}$ is proportional to the identity map. Moreover $M$ is said to be pseudoumbilical in $U \subset M$ or pseudoumbilical if $M$ is pseudoumbilical everywhere on $U$ or everywhere on $M$, respectively.

The mean curvature vector $\bar{H}$ is given by

$$
\bar{H}=H-e_{3}, \quad 2 H=\left(\operatorname{tr} A_{4}\right) e_{4} .
$$

Henceforth we assume that $M$ is mass-symmetric 2-type in $S^{m}$. Then we have the spectral decomposition

$$
x=x_{1}+x_{2}, \quad \Delta x_{1}=\lambda_{1} x_{1}, \quad \Delta x_{2}=\lambda_{2} x_{2}, \quad \lambda_{1}<\lambda_{2} .
$$

Because of the main result in [8] and Theorem 2 in [1] we may assume that $m \geqq 5$. Moreover from our choice of the frame we have $\operatorname{tr} A_{\alpha}=0$ for $\alpha \geqq 5$ and so we may set $h_{11}^{\alpha}=-h_{22}^{\alpha}=\rho_{\alpha}$ and $h_{12}^{\alpha}=h_{21}^{\alpha}=\mu_{\alpha}$. Thus we have

$$
A_{3}=\left(\begin{array}{rr}
-1 & 0 \\
0 & -1
\end{array}\right), \quad A_{4}=\left(\begin{array}{ll}
h_{11}^{4} & h_{12}^{4} \\
h_{12}^{4} & h_{22}^{4}
\end{array}\right) \text { and } A_{\alpha}=\left(\begin{array}{lr}
\rho_{\alpha} & \mu_{\alpha} \\
\mu_{\alpha} & -\rho_{\alpha}
\end{array}\right) \text { for } \alpha \geqq 5 \text {. }
$$

Using Chen's formula ([4], Lemma 4.1, p. 271) and the above expression for $x$ we easily obtain the following necessary conditions for mass-symmetric and 2-type surface in $S^{m}(m \geqq 5)$.

$$
\begin{aligned}
& \left(\operatorname{tr} A_{4}\right)^{2}=2\left(\lambda_{1}+\lambda_{2}\right)-\lambda_{1} \lambda_{2}-4=\left(\lambda_{2}-2\right)\left(2-\lambda_{1}\right), \\
& \sum_{\imath=1}^{2} A_{e_{i} e_{4}} e_{\imath}=0 \\
& \operatorname{tr} A_{4}^{2}+\left|D e_{4}\right|^{2}=\lambda_{2}+\lambda_{2}-2 \\
& \operatorname{tr}\left(A_{4} A_{\alpha}\right)-\operatorname{tr} V \omega_{4 \alpha}+\left\langle D e_{4}, D e_{\alpha}\right\rangle=0, \quad \text { for any } \alpha \geqq 5,
\end{aligned}
$$

where

$$
\left\langle D e_{4}, D e_{\alpha}\right\rangle=\sum_{i=1}^{2}\left\langle D_{e_{i}} e_{4}, D_{e_{i}} e_{\alpha}\right\rangle \text { for any } \alpha \geqq 4
$$

and

$$
\operatorname{tr} \nabla \omega_{4 \alpha}=\sum_{i}\left(\nabla_{e_{i}} \omega_{4 \alpha}\right) e_{\imath}=\sum_{i}\left(e_{i}\left(\omega_{4 \alpha}\left(e_{\imath}\right)\right)-\omega_{4 \alpha}\left(\nabla_{e_{i}} e_{\imath}\right)\right)
$$

LEMMA 1.1. Let $M$ be a mass-symmetric 2-type surface in $S^{m}(m \geqq 5)$. The Weingarten map $A_{4}$ is a Codazzi tensor, that is $A_{4}$ satisfies equation $\left(\bar{\nabla}_{e_{1}} A_{4}\right) e_{2}=$ $\left(\nabla_{e_{2}} A_{4}\right) e_{1}$.

Proof. Since $D_{e_{i} e_{4}}=\Sigma_{\alpha \geq 5} \omega_{4 \alpha}\left(e_{2}\right) e_{\alpha}$, from (1.2) we have

$$
\sum_{\alpha \geq 5}\left(\omega_{4 \alpha}\left(e_{1}\right) \rho_{\alpha}+\omega_{4 \alpha}\left(e_{2}\right) \mu_{\alpha}\right)=0 \text { and } \sum_{\alpha \geq 5}\left(\omega_{4 \alpha}\left(e_{1}\right) \rho_{\alpha}-\omega_{4 \alpha}\left(e_{2}\right) \mu_{\alpha}\right)=0 .
$$


The last equations imply $A_{\substack{D e_{4} \\ e_{1}}} e_{2}=A_{e_{2} e_{4}} e_{1}$ and so from the Codazzi equation

$$
\left(\nabla_{e_{1}} A_{4}\right) e_{2}-\left(\nabla_{e_{2}} A_{4}\right) e_{1}=A_{e_{1} e_{4}} e_{2}-A_{e_{2} e_{4}} e_{1}
$$

we conclude the desired result.

Let $N_{1}(p)$ be the first normal space of $M$ in $S^{m}$ at the point $p \in M$. Considering the linear map $\xi \rightarrow A_{\xi}$ from the normal bundle $\left(T_{p} M\right)^{\perp}$ of $M$ in $S^{m}$ into the bundle whose fiber at $p \in M$ is the space of symmetric linear transformations of $T_{p} M$ we conclude that $\operatorname{dim} N_{1}(p) \leqq 3$. It is obvious that $e_{4}$ belongs to $N_{1}(p)$ for all $p \in M$.

Consider, now, the linear map $L_{p}: T_{p} M \rightarrow\left(T_{p} M\right)^{\perp}$ defined by $L_{p}(X)=D_{X} e_{4}$. We set $d_{p}=\operatorname{dim}\left(L_{p}\left(T_{p} M\right)\right)$. It is obvious that $d_{p} \leqq 2$ everywhere on $M$. In the following let

$$
M_{0}=\left\{p \in M: d_{p}=0\right\}, \quad M_{1}=\left\{p \in M: d_{p}=1\right\} \quad \text { and } \quad M_{2}=\left\{p \in M: d_{p}=2\right\} .
$$

It is clear that $M_{2}$ is an open subset of $M$.

Lemma 1.2. Let $\operatorname{Int}\left(M_{0}\right) \neq \emptyset$. Every component $V_{0}$ of $\operatorname{Int}\left(M_{0}\right)$ lies fully in a totally geodesic $S^{3}$ of $S^{m}$.

Proof. The vectors $D_{e_{1}} e_{4}$ and $D_{e_{2}} e_{4}$ vanish identically on $V_{0}$. Since $M$ is 2-type from equation (1.1) we conclude that $\operatorname{tr} A_{4}=$ const. $\neq 0$. So $V_{0}$ has parallel mean curvature vector in $S^{m}$. According to a result due to B.Y. Chen and S. T. Yau (see for example [3], p. 106), $V_{0}$ lies in a sphere $S^{3}$ of $S^{m}$. Then, the main result in [8] implies that $V_{0}$ is an open portion of a Riemannian product of two circles of different radii in a great sphere $S^{3}$ of $S^{m}$.

Lemma 1.3. Let $\operatorname{Int}\left(M_{1}\right) \neq \emptyset$. On every component $V_{1}$ of $\operatorname{Int}\left(M_{1}\right)$ we may choose $e_{5}, e_{6}, \cdots, e_{\alpha}$ so that $A_{5}=0$ and $A_{\alpha}=0$ for any $\alpha \geqq 8$. Moreover $D_{e_{1}} e_{4}=$ $\omega_{45}\left(e_{1}\right) e_{5}, D_{e_{2}} e_{4}=\omega_{45}\left(e_{2}\right) e_{5}$ and $\omega_{45}^{2}\left(e_{1}\right)+\omega_{45}^{2}\left(e_{2}\right) \neq 0$ everywhere on $V_{1}$.

Proof. The vectors $D_{e_{1}} e_{4}, D_{e_{2}} e_{4}$ are linearly dependent everywhere on $V_{1}$ and at least one of them is nonzero. Denote by $e_{5}$ its common unit direction. Then we have

$$
D_{e_{1}} e_{4}=\omega_{45}\left(e_{1}\right) e_{5} \text { and } D_{e_{2}} e_{4}=\omega_{45}\left(e_{2}\right) e_{5}
$$

and $\omega_{45}^{2}\left(e_{1}\right)+\omega_{45}^{2}\left(e_{2}\right) \neq 0$, everywhere on $V_{1}$. In this case from equation (1.2) we find

$$
\omega_{45}\left(e_{1}\right) \rho_{5}+\omega_{45}\left(e_{2}\right) \mu_{5}=0 \text { and } \omega_{45}\left(e_{1}\right) \mu_{5}-\omega_{45}\left(e_{2}\right) \rho_{5}=0
$$

from which we obtain $\mu_{5}=\rho_{5}=0$. Thus $A_{5}=0$, that is $e_{5}$ nowhere belongs to the first normal space. Then we choose $e_{6}, e_{7}$ so that $e_{6}, e_{7} \in N_{1}$ and thus $e_{\alpha} \notin N_{1}$ for any $\alpha \geqq 8$. 
LEMMA 1.4. Let $M_{2} \neq \emptyset$. On every component $V_{2}$ of $M_{2}$ we may choose $e_{5}$, $e_{6}$ so that

$$
D_{e_{1}} e_{4}=\omega_{45}\left(e_{1}\right) e_{5}, \quad D_{e_{2} e_{4}}=\omega_{45}\left(e_{2}\right) e_{5}+\omega_{46}\left(e_{2}\right) e_{6} \quad \text { and } \omega_{45}\left(e_{1}\right) \cdot \omega_{46}\left(e_{2}\right) \neq 0
$$

everywhere on $V_{2}$. Moreover we may suppose that $V_{2}$ consists in open neighbourhoods $V_{21}, V_{22}$ such that one of the following holds

$$
\begin{array}{ll}
A_{5} \neq 0, A_{6} \neq 0 \quad \text { everywhere on } V_{21} \\
A_{5}=0 \text { and } A_{6}=0 \text { everywhere on } V_{22} .
\end{array}
$$

Proof. Because the vectors $D_{e_{1}} e_{4}, D_{e_{2}} e_{4}$ are linearly independent everywhere on $V_{2}$, by Gram-Schmidt orthogonalization process the first part of Lemma is established. For the proof of the second part it is necessary to prove that there is no open set of $V_{2}$ where $A_{5} \neq 0$ and $A_{6}=0$ or $A_{5}=0$ and $A_{6} \neq 0$. In fact if such an open set there exists, then from equation (1.2) we have

or

$$
\rho_{5} \omega_{45}\left(e_{1}\right)+\mu_{5} \omega_{45}\left(e_{2}\right)=0, \quad \mu_{5} \omega_{45}\left(e_{1}\right)-\rho_{5} \omega_{45}\left(e_{2}\right)=0
$$

$$
\omega_{46}\left(e_{2}\right) \mu_{6}=0, \quad \omega_{46}\left(e_{2}\right) \rho_{6}=0
$$

which are impossible.

Remark 1.5. In the following we refer quite often to neighbourhoods such as $V_{0}, V_{1}, V_{2}, V_{21}$ and $V_{22}$ which are defined in the above Lemmas.

In order to state another lemma which is useful in the proof of global results, we restrict to a nowhere pseudoumbilical neighbourhood $U$ of $M$. In this situation we may choose $e_{1}, e_{2}$ as the eigenvectors of $A_{4}$ with corresponding eigenvalues $k_{1}, k_{2}\left(k_{1}>k_{2}\right)$. In that case we have, $k_{1}+k_{2}=\operatorname{tr} A_{4}=$ const. $\neq 0$, as it follows from equation (1.1) and because $M$ is of 2-type.

LEMMA 1.6. Let $M$ be a mass-symmetric 2-type surface in $S^{m}$. In a nowhere pseudoumbilical neighbourhood $U$ the curvature form $\omega_{12}$ and the Gaussian curvature $K$ are given by

$$
\begin{aligned}
& \omega_{12}=\frac{1}{2} \frac{e_{2}\left(k_{1}-k_{2}\right)}{k_{1}-k_{2}} \omega_{1}-\frac{1}{2} \frac{e_{1}\left(k_{1}-k_{2}\right)}{k_{1}-k_{2}} \omega_{2} \\
& K=-\frac{1}{2} \Delta \log \left(k_{1}-k_{2}\right) .
\end{aligned}
$$

Proof. From our choice of the frame we have $\omega_{i 4}=k_{i} \omega_{i}, k_{1}+k_{2}=$ const. and $\omega_{1 \alpha}=\rho_{\alpha} \omega_{1}+\mu_{\alpha} \omega_{2}, \omega_{2 \alpha}=\mu_{\alpha} \omega_{1}-\rho_{\alpha} \omega_{2}$. Because of (1.6) we find

and thus

$$
\sum_{\alpha \geq 5} \omega_{1 \alpha} \wedge \omega_{\alpha 4}=0 \text { and } \sum_{\alpha \geq 5} \omega_{2 \alpha} \wedge \omega_{\alpha 4}=0
$$




$$
d \omega_{14}=\omega_{12} \wedge \omega_{24}, \quad d \omega_{24}=\omega_{21} \wedge \omega_{14} .
$$

The last equations imply (1.6). By using (1.6) and the Gauss equation

$$
K=e_{2}\left(\omega_{12}\left(e_{1}\right)\right)-e_{1}\left(\omega_{12}\left(e_{2}\right)\right)-\left(\omega_{12}\left(e_{1}\right)\right)^{2}-\left(\omega_{12}\left(e_{2}\right)\right)^{2}
$$

we conclude (1.7).

\section{Local results}

Let $M$ be a mass-symmetric 2-type surface in $S^{m}, m \geqq 5$. In this section we prove some local results. in $S^{m}$.

PROPOSITION 2.1. There is no neighbourhood of the form $V_{0}$ which lies fully

Proof. This is a immediate consequence of Lemma 1.2.

Proposition 2.2. Let $M$ be a mass-symmetric 2-type surface in $S^{m}, m \geqq 5$. If $M$ has a neighbourhood of the form $V_{1}$ then $M$ is nowhere pseudoumbilical in an open set of $V_{1}$ and $V_{1}$ is flat.

Proof. By Lemma 1.3 we may set $\omega_{45}=\beta_{1} \omega_{1}+\beta_{2} \omega_{2}$, where $\beta_{1}^{2}+\beta_{2}^{2} \neq 0$. By exterior differentiation of $\omega_{46}=0$ and $\omega_{47}=0$ we get respectively

and

$$
-\beta_{2} \omega_{56}\left(e_{1}\right)+\beta_{1} \omega_{56}\left(e_{2}\right)=\left(k_{1}-k_{2}\right) \mu_{6}
$$

$$
-\beta_{2} \omega_{57}\left(e_{1}\right)+\beta_{1} \omega_{57}\left(e_{2}\right)=\left(k_{1}-k_{2}\right) \mu_{7} .
$$

Moreover (1.4) for $\alpha=6$ and $\alpha=7$ becomes respectively

and

$$
\beta_{1} \omega_{56}\left(e_{1}\right)+\beta_{2} \omega_{56}\left(e_{2}\right)=\left(k_{1}-k_{2}\right) \rho_{6}
$$

Hence we find

$$
\beta_{1} \omega_{57}\left(e_{1}\right)+\beta_{2} \omega_{57}\left(e_{2}\right)=\left(k_{1}-k_{2}\right) \rho_{7} .
$$

$$
\begin{aligned}
& \omega_{56}\left(e_{1}\right)=\frac{k_{1}-k_{2}}{\beta_{1}^{2}+\beta_{2}^{2}}\left(\beta_{1} \rho_{6}-\beta_{2} \mu_{6}\right) \\
& \omega_{56}\left(e_{2}\right)=\frac{k_{1}-k_{2}}{\beta_{1}^{2}+\beta_{2}^{2}}\left(\beta_{1} \mu_{6}+\beta_{2} \rho_{6}\right) \\
& \omega_{57}\left(e_{1}\right)=\frac{k_{1}-k_{2}}{\beta_{1}^{2}+\beta_{2}^{2}}\left(\beta_{1} \rho_{7}-\beta_{2} \mu_{7}\right) \\
& \omega_{57}\left(e_{2}\right)=\frac{k_{1}-k_{2}}{\beta_{1}^{2}+\beta_{2}^{2}}\left(\beta_{1} \mu_{7}+\beta_{2} \rho_{7}\right) .
\end{aligned}
$$

Differentiation of $\omega_{15}=\omega_{25}=0$ gives 


$$
\begin{array}{r}
k_{1} \beta_{2}+\rho_{6} \omega_{65}\left(e_{2}\right)-\mu_{6} \omega_{65}\left(e_{1}\right)+\rho_{7} \omega_{75}\left(e_{2}\right)-\mu_{7} \omega_{75}\left(e_{1}\right)=0 \\
-\beta_{1} k_{2}+\mu_{6} \omega_{65}\left(e_{2}\right)+\rho_{6} \omega_{65}\left(e_{1}\right)+\mu_{7} \omega_{75}\left(e_{2}\right)+\rho_{7} \omega_{75}\left(e_{1}\right)=0 .
\end{array}
$$

Substituting $\omega_{65}\left(e_{1}\right), \omega_{65}\left(e_{2}\right), \omega_{75}\left(e_{1}\right), \omega_{75}\left(e_{2}\right)$ into the last two equations we get respectively

$$
\begin{aligned}
& k_{1} \beta_{2}-\beta_{2}\left(\rho_{6}^{2}+\mu_{6}^{2}+\rho_{7}^{2}+\mu_{7}^{2}\right) \frac{k_{1}-k_{2}}{\beta_{1}^{2}+\beta_{2}^{2}}=0 \\
& k_{2} \beta_{1}+\beta_{1}\left(\rho_{6}^{2}+\mu_{6}^{2}+\rho_{7}^{2}+\mu_{7}^{2}\right) \frac{k_{1}-k_{2}}{\beta_{1}^{2}+\beta_{2}^{2}}=0 .
\end{aligned}
$$

Because $k_{1}+k_{2}$ is a nonzero constant from the last equations we infer $\beta_{1} \beta_{2}=0$. Moreover from these equations we conclude that $M$ is nowhere pseudoumbilical in an open set of $V_{1}$. Without loss of generality we can suppose that $\beta_{2}=0$; then $\omega_{45}=\beta_{1} \omega_{1}$ and by exterior differentiation we find

Using (1.6) we get

$$
\omega_{12}\left(e_{1}\right)=\frac{e_{2}\left(\beta_{1}\right)}{\beta_{1}} \text {. }
$$

$$
e_{2}\left(\frac{\beta_{1}^{2}}{k_{1}-k_{2}}\right)=0 \text {. }
$$

Furthermore (1.4) for $\alpha=5$ and taking account that $A_{5}=0$, becomes

$$
e_{1}\left(\beta_{1}\right)+\beta_{1} \omega_{12}\left(e_{2}\right)=0
$$

from which by using (1.6) we obtain

$$
e_{1}\left(\frac{\beta_{1}^{2}}{k_{1}-k_{2}}\right)=0 \text {. }
$$

Hence there is a constant $d$ such that $\beta_{1}^{2}=d\left(k_{1}-k_{2}\right)$. Now, using (1.3) and (1.1), we easily, deduce that $k_{1}, k_{2}, \beta_{1}$ are constant. The flatness of $V_{1}$ follows now from Lemma 1.6.

PROPOSITION 2.3. Let $M$ be a mass-symmetric 2-type surface in $S^{m}, m \geqq 6$. If $M$ has a neighbourhood of form $V_{21}$ then this lies fully in a totally geodesic sphere $S^{5}$ of $S^{m}$.

Proof. In $V_{21}$ by using Lemma 1.4 we set $\omega_{45}=\beta_{1} \omega_{1}+\beta_{2} \omega_{2}$ and $\omega_{46}=\gamma_{2} \omega_{2}$ where $\beta_{1} \gamma_{2} \neq 0$, everywhere in $V_{21}$. Moreover $\mu_{5}^{2}+\rho_{5}^{2} \neq 0, \mu_{6}^{2}+\rho_{6}^{2} \neq 0$ and $A_{\alpha}=0$ for any $\alpha \geqq 7$. Since $\omega_{1 \alpha}=\omega_{2 \alpha}=0$ for any $\alpha \geqq 7$, (1.2) implies

$$
\begin{aligned}
& \beta_{1} \rho_{5}+\beta_{2} \mu_{5}+\gamma_{2} \mu_{6}=0 \\
& \beta_{1} \mu_{5}-\beta_{2} \rho_{5}-\gamma_{2} \rho_{6}=0 .
\end{aligned}
$$

Because $\omega_{4 \alpha}=0$ for any $\alpha \geqq 7$, relation (1.4) implies 


$$
\beta_{1} \omega_{\alpha 5}\left(e_{1}\right)+\beta_{2} \omega_{\alpha 5}\left(e_{1}\right)+\gamma_{1} \omega_{\alpha 6}\left(e_{2}\right)+\gamma_{2} \omega_{\alpha 6}\left(e_{2}\right)=0, \quad \alpha \geqq 7 .
$$

On the other hand by exterior differentiation of $\omega_{4 \alpha}=\omega_{1 \alpha}=\omega_{2 \alpha}=0, \alpha \geqq 7$ one finds

$$
\begin{aligned}
& \beta_{1} \omega_{\alpha 5}\left(e_{2}\right)-\beta_{2} \omega_{\alpha 5}\left(e_{1}\right)-\gamma_{2} \omega_{\alpha 6}\left(e_{1}\right)=0, \\
& \rho_{5} \omega_{\alpha 5}\left(e_{1}\right)+\mu_{5} \omega_{\alpha 5}\left(e_{2}\right)+\rho_{6} \omega_{\alpha 6}\left(e_{1}\right)+\mu_{6} \omega_{\alpha 6}\left(e_{2}\right)=0, \\
& -\mu_{5} \omega_{\alpha 5}\left(e_{1}\right)+\rho_{5} \omega_{\alpha 5}\left(e_{2}\right)-\mu_{6} \omega_{\alpha 6}\left(e_{1}\right)+\rho_{6} \omega_{\alpha 6}\left(e_{2}\right)=0 .
\end{aligned}
$$

We consider (2.3), (2.4), (2.5) and (2.6) as a linear system with respect to $\omega_{\alpha 5}\left(e_{1}\right)$, $\omega_{\alpha 5}\left(e_{2}\right), \omega_{\alpha 6}\left(e_{1}\right), \omega_{\alpha 6}\left(e_{2}\right)$. Denote by $T$ the determinant of the system. We claim that $T$ is everywhere positive on $V_{21}$. In fact by computation we find

$$
T=\left(\beta_{1}^{2}+\beta_{2}^{2}\right)\left(\rho_{6}^{2}+\mu_{6}^{2}\right)+\gamma_{2}^{2}\left(\rho_{5}^{2}+\mu_{5}^{2}\right)+2 \beta_{1} \gamma_{2}\left(\mu_{5} \rho_{6}-\rho_{5} \mu_{6}\right)-2 \beta_{2} \gamma_{2}\left(\rho_{5} \rho_{6}+\mu_{5} \mu_{6}\right),
$$

which by using (2.1) and (2.2) becomes

$$
T=\left(\beta_{1}^{2}+\beta_{2}^{2}\right)\left(\rho_{6}^{2}+\mu_{6}^{2}\right)+\gamma_{2}^{2}\left(\rho_{5}^{2}+\mu_{5}^{2}\right)+2 \gamma_{2}^{2}\left(\mu_{6}^{2}+\rho_{6}^{2}\right),
$$

which proves the claim. So, the system yields $\omega_{5 \alpha}=\omega_{6 \alpha}=0$, for any $\alpha \geqq 7$. But $\omega_{4 \alpha}=0$, for any $\alpha \geqq 7$. It follows that the first normal space which is spanned by $e_{4}, e_{5}, e_{6}$ is parallel in the normal bundle of $M$ in $S^{m}$. So, the codimension of $V_{21}$ is reduced to 3 by Erbacher's Theorem [6] and $V_{21}$ lies in a totally geodesic sphere $S^{5}$ of $S^{m}$.

Since in the following we shall apply at times the result of Y. Miyata [12] the following lemma is necessary.

LEMMA 2.4. Let $M$ be a mass-symmetric 2-type surface in $S^{m}$ Then $0<\lambda_{1}<2<\lambda_{2}$.

Proof. Taking account of (1.1) one finds

$$
k_{1}^{2}+k_{2}^{2}=\frac{\left(k_{1}+k_{2}\right)^{2}}{2}+\frac{\left(k_{1}-k_{2}\right)^{2}}{2}=\lambda_{1}+\lambda_{2}-\frac{\lambda_{1} \lambda_{2}}{2}-2+\frac{\left(k_{1}-k_{2}\right)^{2}}{2}
$$

and because of (1.3) we have

$$
\frac{\left(k_{1}-k_{2}\right)^{2}}{2}+\left|D e_{4}\right|^{2}=\frac{\lambda_{1} \lambda_{2}}{2},
$$

which proves the assertion, by using (1.1).

Now we are able to prove the following.

THEOREM 2.5. Let $M$ be a mass-symmetric 2-type surface in $S^{m}, m \geqq 5$. If $\operatorname{dim} N_{1} \leqq 2$ and $M$ lies fully in $S^{m}$ then $m$ is odd unless $M$ is pseudoumbilical.

Proof. If $m=5$ there is nothing to prove. So, we may suppose $m \geqq 6$. Because of Propositions 2.1 and $2.3 \mathrm{M}$ cannot has neighbourhoods of form $V_{0}$, 
$V_{21}$ which are fully in $S^{m}$ with $m \geqq 6$. So, there is neighbourhood of form $V_{1}$ or of form $V_{22}$ which lies fully in $S^{m}$ with $m \geqq 6$. In the first case $V_{1}$ must be flat by Proposition 2.2. But then a result due to Y. Miyata ([12], Theorem and its Corollary) implies that $m$ is odd.

From now on we suppose that $M$ has a neighbourhood of form $V_{22}$ nowhere pseudoumbilical which lies fully in $S^{m}$ with $m \geqq 6$. Moreover, because of $\operatorname{dim} N_{1} \leqq 2$ and $e_{4} \in N_{1}$ we may assume that $\rho_{8}=\mu_{8}=0$ everywhere on $V_{22}$. We set $\omega_{45}=\beta_{1} \omega_{1}+\beta_{2} \omega_{2}$ and $\omega_{46}=\gamma_{2} \omega_{2}$, where $\beta_{1} \gamma_{2} \neq 0$ by Lemma 1.4. Taking exterior differentiation of $\omega_{15}=\omega_{25}=0$, we get

$$
\begin{aligned}
& \rho_{7} \omega_{75}\left(e_{1}\right)+\mu_{7} \omega_{75}\left(e_{2}\right)=\beta_{1} k_{2} \\
& -\mu_{7} \omega_{75}\left(e_{1}\right)+\rho_{7} \omega_{75}\left(e_{2}\right)=-k_{1} \beta_{2} .
\end{aligned}
$$

Similarly, from $\omega_{16}=\omega_{26}=0$ we obtain

$$
\begin{aligned}
& \rho_{7} \omega_{76}\left(e_{1}\right)+\mu_{7} \omega_{76}\left(e_{2}\right)=0 \\
& -\mu_{7} \omega_{76}\left(e_{1}\right)+\rho_{7} \omega_{76}\left(e_{2}\right)=-k_{1} \gamma_{2} .
\end{aligned}
$$

We claim that $\rho_{7}^{2}+\mu_{7}^{2} \neq 0$ on an open subset of $V_{22}$. Assume in the contrary that $\rho_{7}^{2}+\mu_{7}^{2}=0$ in an open subset $V \subset V_{22}$. Then it follows from the above relations that $k_{1} \cdot k_{2}=0$ and since $k_{1}+k_{2}=$ const. we would have from Lemma 1.6 that $K=0$. On the other hand from Gauss equation we find $K=1$ which is impossible. Therefore we get

$$
\begin{aligned}
& \omega_{75}\left(e_{1}\right)=\frac{1}{\rho_{7}^{2}+\mu_{7}^{2}}\left(k_{2} \beta_{1} \rho_{7}+k_{1} \beta_{2} \mu_{7}\right) \\
& \omega_{75}\left(e_{2}\right)=\frac{1}{\rho_{7}^{2}+\mu_{7}^{2}}\left(-k_{1} \beta_{2} \rho_{7}+k_{2} \beta_{1} \mu_{7}\right) \\
& \omega_{76}\left(e_{1}\right)=\frac{1}{\rho_{7}^{2}+\mu_{7}^{2}} k_{1} \gamma_{2} \mu_{7} \\
& \omega_{76}\left(e_{2}\right)=-\frac{1}{\rho_{7}^{2}+\mu_{7}^{2}} k_{1} \gamma_{2} \rho_{7} .
\end{aligned}
$$

Differentiating $\omega_{47}=0$ we get

$$
\left(k_{2}-k_{1}\right) \mu_{7}+\beta_{1} \omega_{57}\left(e_{2}\right)-\beta_{2} \omega_{57}\left(e_{1}\right)-\gamma_{2} \omega_{67}\left(e_{1}\right)=0 .
$$

Moreover (1.4) for $\alpha=7$ becomes

$$
\left(k_{1}-k_{2}\right) \rho_{7}+\beta_{1} \omega_{75}\left(e_{1}\right)+\beta_{2} \omega_{75}\left(e_{2}\right)+\gamma_{2} \omega_{76}\left(e_{2}\right)=0 .
$$

Substituting $\omega_{75}\left(e_{1}\right), \omega_{75}\left(e_{2}\right), \omega_{76}\left(e_{1}\right)$ and $\omega_{76}\left(e_{2}\right)$ into the last equations we obtain

$$
\left(k_{1}-k_{2}\right) \mu_{7}+\frac{1}{\rho_{7}^{2}+\mu_{7}^{2}}\left(-k_{1} \beta_{1} \beta_{2} \rho_{7}+k_{2} \beta_{1}^{2} \mu_{7}-k_{2} \beta_{1} \beta_{2} \rho_{7}-k_{1} \beta_{2}^{2} \mu_{7}-k_{1} \gamma_{2}^{2} \mu_{7}\right)=0
$$




$$
\left(k_{1}-k_{2}\right) \rho_{7}+\frac{1}{\rho_{7}^{2}+\mu_{7}^{2}}\left(k_{2} \beta_{1}^{2} \rho_{7}+k_{1} \beta_{1} \beta_{2} \mu_{7}-k_{1} \beta_{2}^{2} \rho_{7}-k_{2} \beta_{1} \beta_{2} \mu_{7}-k_{1} \gamma_{2}^{2} \mu_{7}\right)=0 .
$$

Eliminating the terms $\left(k_{1}-k_{2}\right) \mu_{7}$ and $\left(k_{1}-k_{2}\right) \rho_{7}$ from (2.7) and (2.8) we find $\beta_{1} \beta_{2}=0$ and thus $\beta_{2}=0$. Hence (2.7) and (2.9) give

$$
k_{1}-k_{2}+\frac{k_{2} \beta_{1}^{2}-k_{1} \gamma_{2}^{2}}{\rho_{7}^{2}+\mu_{7}^{2}}=0 .
$$

Differentiating $\omega_{45}=\beta_{1} \omega_{1}$ we obtain

$$
-e_{2}\left(\beta_{1}\right)+\beta_{1} \omega_{12}\left(e_{1}\right)=\gamma_{2} \omega_{56}\left(e_{1}\right)
$$

and using (1.6) we get

$$
e_{2}\left(\frac{\beta_{1}^{2}}{k_{1}-k_{2}}\right)=-\frac{2 \beta_{1} \gamma_{2}}{k_{1}-k_{2}} \omega_{56}\left(e_{1}\right) .
$$

On the other hand (1.4) for $\alpha=6$ on account of $\beta_{2}=0$ and (1.6) becomes

$$
e_{2}\left(\frac{\gamma_{2}^{2}}{k_{1}-k_{2}}\right)=\frac{2 \beta_{1} \gamma_{2}}{k_{1}-k_{2}} \omega_{65}\left(e_{1}\right) \text {. }
$$

Therefore

$$
e_{2}\left(\frac{\beta_{1}^{2}-\gamma_{2}^{2}}{k_{1}-k_{2}}\right)=0
$$

By a similar argument on $\omega_{46}=\gamma_{2} \omega_{2},(1.4)$ for $\alpha=5$ and (1.6) we also have

$$
e_{1}\left(\frac{\beta_{1}^{2}-\gamma_{2}^{2}}{k_{1}-k_{2}}\right)=0 \text {. }
$$

Consequently,

$$
\beta_{1}^{2}-\gamma_{2}^{2}=d\left(k_{1}-k_{2}\right)
$$

where $d$ is a constant. Eliminating $\beta_{1}$ and $\gamma_{2}$ from (1.3), (2.9) and (2.10) we get

$$
2\left(\rho_{7}^{2}+\mu_{7}^{2}\right)+k_{1}^{2}+k_{2}^{2}=\lambda_{1}+\lambda_{2}-2-d\left(k_{1}+k_{2}\right)
$$

which implies that the Gaussian curvature of $V_{22}$ is constant, since $k_{1}+k_{2}=$ const. Using again the result of Y. Miyata [12] we infer that $m$ is odd and the proof is completed.

COROLlARY 2.6. Let $M$ be a mass-symmetric 2-type surface in $S^{m}$. If $M$ has flat normal connection and lies fully in $S^{m}$ then $m$ is odd unless $M$ is pseudoumbilical.

Proof. It is obvious that the flatness of the normal connection implies that $\operatorname{dim} N_{1} \leqq 2$. 


\section{Global results}

In this section we give two global results.

THEOREM 3.1. Let $M$ be a complete mass-symmetric 2-type surface in $S^{m}$. If $M$ has non-negative Gaussian curvature and lies fully in $S^{m}$ then it is either pseudoumbilical or flat. In the last case $m$ is odd.

Proof. Since $M$ is complete with Gaussian curvature $K \geqq 0$ by a theorem of A. Huber [10] we know that $M$ is either compact or parabolic. At first we assume that $M$ is compact. If $K$ is not identically zero then $M$ is homeomorphic to $S^{2}$. Therefore by a result of M. Kotani [11] $m$ is odd. If $K=0$ identically, then the conclusion follows from the result of Y. Miyata [12].

Now assume that $M$ is non-compact and parabolic. We know from Lemma 1.1 that $A_{4}$ is a Codazzi tensor with constant trace, by using a result of $\mathrm{B}$. Wegner [13, Satz 1] we get

$$
-\frac{1}{2} \Delta \operatorname{tr} A_{4}^{2}=K\left(k_{1}-k_{2}\right)^{2}+\left|\nabla A_{4}\right|^{2} .
$$

Since $K \geqq 0$ the above equation implies that $\operatorname{tr} A_{4}^{2}$ is a subharmonic function on $M$. On the other hand from Gauss equation we obtain

$$
\operatorname{tr} A_{4}^{2} \leqq 4\left(|H|^{2}+1\right) \text {. }
$$

Therefore $\operatorname{tr} A_{4}^{2}$ is bounded from above. Consequently $\operatorname{tr} A_{4}^{2}$ is a constant since $M$ is parabolic and thus $k_{1}, k_{2}$ are constants. It follows then from (3.1) that $K\left(k_{1}-k_{2}\right)^{2}=0$. So $M$ is either pseudoumbilical or nowhere pseudoumbilical. In the last case using Lemma 1.1 we infer that $M$ is flat and applying once more the result of Y. Miyata [12] we conclude that $m$ is odd.

THEOREM 3.2. Let $M$ be a compact mass-symmetric 2-type surface in $S^{m}$ with non-positive Gauss curvature $K$. If $M$ is nowhere pseudoumbilical and lies fully in $S^{m}$ then it is flat and $m$ is odd.

Proof. From our assumption and Lemma 1.1 we have $\Delta \log \left(k_{1}-k_{2}\right) \geqq 0$ on $M$. By the compactness of $M$ and the maximum principle we obtain $k_{1}-k_{2}=$ const. Since $k_{1}+k_{2}=$ const. we get that $k_{1}, k_{2}$ are constant on $M$. Appealing again to Lemma 1.1 we deduce $K=0$ on $M$ and so on.

\section{A non-existence theorem}

In this paragraph we prove the following non-existence result.

THEOREM 4.1. There exist no mass-symmetric 2-type surfaces which lie fully in $S^{6}$. 
Proof. By the proof of Theorem 2.5 it is enough to consider the case when there is a neighbourhood of the form $V_{22}$ where $M$ is pseudoumbilical. By Lemma 1.4 we may suppose that

$$
A_{4}=\left(\begin{array}{cc}
k_{1} & 0 \\
0 & k_{1}
\end{array}\right), \quad A_{5}=A_{6}=0, \quad A_{7}=\left(\begin{array}{cr}
\rho_{7} & 0 \\
0 & -\rho_{7}
\end{array}\right)
$$

and $\omega_{45}=\beta_{1} \omega_{1}+\beta_{2} \omega_{2}, \omega_{46}=\gamma_{2} \omega_{2}$ where $\beta_{1} \gamma_{2} \neq 0$ everywhere on $V_{22}$ and $k_{1}$ is a nonzero constant. Differentiating $\omega_{15}=0, \omega_{25}=0, \omega_{16}=0, \omega_{26}=0$ we get respectively

$$
\begin{aligned}
& \rho_{7} \omega_{75}\left(e_{2}\right)=-k_{1} \beta_{2} \\
& \rho_{7} \omega_{75}\left(e_{1}\right)=k_{1} \beta_{1} \\
& \rho_{7} \omega_{76}\left(e_{2}\right)=-k_{1} \gamma_{2} \\
& \rho_{7} \omega_{76}\left(e_{1}\right)=0 .
\end{aligned}
$$

It is obvious that $\rho_{7}$ is nonzere everywhere on $V_{22}$.

Taking exterior differentiation of $\omega_{47}=0$ we obtain

$$
\beta_{1} \omega_{75}\left(e_{2}\right)-\beta_{2} \omega_{75}\left(e_{1}\right)-\gamma_{2} \omega_{76}\left(e_{1}\right)=0 .
$$

Furthermore (1.4) for $\alpha=7$ becomes

$$
\beta_{1} \omega_{75}\left(e_{1}\right)+\beta_{2} \omega_{75}\left(e_{2}\right)+\gamma_{2} \omega_{76}\left(e_{2}\right)=0 .
$$

Substituting $\omega_{75}\left(e_{1}\right), \omega_{75}\left(e_{2}\right), \omega_{76}\left(e_{1}\right), \omega_{76}\left(e_{2}\right)$ from (4.1), (4.2), (4.3) and (4.4) into (4.5) and (4.6) we get respectively

$$
\beta_{1} \beta_{2}=0 \text { and } \beta_{1}^{2}-\beta_{2}^{2}=0 .
$$

From the first of the above equations we conclude that $\beta_{2}=0$ and so $\beta_{1}^{2}=\gamma_{2}^{2}$. Then (1.3) implies that $\beta_{1}$ and $\gamma_{2}$ are nonzero constants.

Taking, now, exterior differentiation of $\omega_{45}=\beta_{1} \omega_{1}$ and $\omega_{46}=\gamma_{2} \omega_{2}$ we get $\omega_{56}= \pm \omega_{12}$ and so, using $d \omega_{12}=-K \omega_{1} \wedge \omega_{2}$, we obtain

$$
K= \pm \beta_{1}^{2}\left(\frac{k_{1}^{2}}{\rho_{7}^{2}}-1\right) \text {. }
$$

On the other hand

$$
K=1+k_{1}^{2}-\rho_{7}^{2} .
$$

Comparing these equations we deduce that $\rho_{7}$ is a constant and moreover $K$ is a nonzero constant. So we have a mass-symmetric 2-type surface with a constant Gauss curvature which lies fully in $S^{6}$ a contradiction of Y. Miyata [12] result. This completes the proof. 


\section{REFERENCES}

[1] M. BArros AND B. Y. Chen, Stationary 2-type surfaces in a hypersphere, J. Math. Soc. Japan, 39 (1987), 627-648.

[2] R. BRyant, Minimal surfaces of constant curvature in $S^{n}$, Trans. Amer. Math. Soc., 290 (1985), 259-271.

[ 3 ] B. Y. Chen, Geometry of Submanifolds, Marcel Dekker, New York, 1973.

[4] B.Y. Chen, Total Mean Curvature and Submanifolds of Finite Type, World Scien tific, Singapore, 1984.

[5] B. Y. CHEN, Some open problems and conjectures on submanifolds of finite type, Soochow J. Math., 17 (1991), 169-188.

[6] J. ERBACHER, Reduction of the codimension of an isometric immersion, J. Differential Geom., 5 (1971), 333-340.

[7] O.J. GARAY, Spherical Chen surfaces which are mass-symmetric and of 2-type, J. Geom., 33 (1988), 39-52.

[8] Th. Hasanis and TH. Vlachos, A local classification of 2-type surfaces in $S^{3}$, Proc. Amer. Math. Soc., 112 (1991), 533-538.

[9] Th. Hasanis and Th. Vlachos, Spherical 2-type hypersurfaces, J. Geom., 40 (1991), 82-94.

[10] A. Huber, On subharmonic functions and differential geometry in the large, Comment. Math. Helv., 32 (1957), 13-72.

[11] M. Kotani, A decomposition theorem of 2-type immersions, Nagoya Math. J., 118 (1990), 55-64.

[12] Y. Miyata, 2-type surfaces of constant curvature in $S^{n}$, Tokyo J. Math., 11 (1988), 157-204.

[13] B. WEGNER, Codazzi-Tensoren und Kennzeichnungen sphärischer Immersionen, J. Differential Geom., 9 (1974), 61-70.

[14] T. TAKAHASHI, Minimal immersions of Riemannian manifolds, J. Math. Soc. Japan, 18 (1966), 380-385.

Department of Mathematics

UNIVERSITY OF IOANNINA

IOANNINA 45110, GREECE

Department of Mathematics

UNIVERSITY OF CRETE

IRAKLION 71409, GREECE 